\title{
It's about politics, stupid! Warum das internationale Strafrecht die Friedensforschung braucht
}

\author{
Plädoyer eines Juristen
}

\section{Einleitung}

Im Juli 1998 wurde auf der Bevollmächtigtenkonferenz von Rom eine neue internationale Institution ins Leben gerufen. Vier Jahre später nahm der Internationale Strafgerichtshof (IStGH) in Den Haag, Niederlande, seine Arbeit auf. Ein weiteres Jahrzehnt ist mittlerweile vergangen, das Verfahren gegen den kongolesischen Rebellenführer Thomas Lubanga Diylo steuert auf einen erfolgreichen Abschluss zu und spätestens seit dem Erlass der Haftbefehle gegen den mittlerweile verstorbenen libyschen Revolutionsführer Muhammar Gaddafi und seine Entourage ist der IStGH nun auch der interessierten bundesrepublikanischen Öffentlichkeit ein Begriff. Ob ZEIT, SPIEGEL, Süddeutsche Zeitung oder FAZ: (selbst) die deutschen Printmedien fangen an über eine Institution zu berichten, die sich ausweislich der Präambel ihres Gründungsstatuts zum Ziel gesetzt hat, »der Straflosigkeit der Täter ein Ende zu setzen und so zur Verhütung solcher Verbrechen beizutragen«. So sollen Verbrechen, wie Völkermord, Verbrechen gegen die Menschlichkeit und Kriegsverbrechen, geahndet werden, denen aufgrund ihrer zumeist enormen Brutalität, ihres Ausmaßes und der oftmals zu verzeichnenden Beteiligung der Staatsorgane ein enormes friedensbedrohliches Potential zugeschrieben wird.

Angesichts der wachsenden Bedeutung des internationalen Strafrechts, der internationalen Strafverfolgung und der Rezeption der Thematik in der Presse verwundert es, dass die deutschen friedenswissenschaftlichen Fachmedien einen großen Bogen um das Thema zu machen scheinen. Dabei ist eine wissenschaftliche Auseinandersetzung mit dem Wirken des Haager Gerichts zu bedeutend, um das Feld alleine den Juristen zu überlassen. Phänomene der Verrechtlichung internationaler Konfliktbewältigung und der Politisierung internationaler Rechtsprechung bieten Anlass genug, einen interdisziplinären Ansatz ins Auge zu fassen und die Tätigkeit des IStGH (friedens-)wissenschaftlich zu begleiten.

In diesem Beitrag soll zunächst ein kurzer Überblick über den Stand der wissenschaftlichen Auseinandersetzung in der deutschen Friedensforschung gegeben werden. Dafür werden exemplarisch die relevanten Beiträge in den führenden Fach- 
zeitschriften ausgewertet. Im Anschluss sollen wichtige Fragestellungen im Themenkomplex »Internationales Strafrecht« und, in der hier gebotenen Kürze, die Möglichkeiten und der Bedarf für friedenswissenschaftliche Durchdringung aufgezeigt werden.

\section{Rezeption der Materie in den Fachmedien}

Folgende periodisch erscheinende Publikationen wurden für den Zeitraum 2006-2011 untersucht: Aus Politik und Zeitgeschichte, Blätter für deutsche und internationale Politik, Friedensgutachten, Die Friedenswarte, Hamburger Beiträge und Informationen zur Friedensforschung, HSFK-Standpunkte, Internationale Politik, Internationale Politik und Gesellschaft, Mittelweg 36, Politische Vierteljahresschrift, Sicherheit und Frieden, Vereinte Nationen, Welttrends, Wissenschaft \& Frieden, Zeitschrift für Internationale Beziehungen, Zeitschrift für Politik, Zeitschrift für Politikwissenschaft. Die Auswahl erhebt keinen Anspruch abschließend zu sein, dürfte jedoch als repräsentativ anzusehen sein. Es wurde untersucht, ob und inwieweit die Tätigkeit internationaler Strafverfolgungsbehörden in Fachzeitschriften aufgegriffen wurde und wie sich die Beiträge der Thematik nähern.

Die Ergebnisse sind leider ernüchternd: Die Anzahl wissenschaftlicher Beiträge mit Bezug zum Völkerstrafrecht bewegt sich im niedrigen zweistelligen Bereich. Zieht man zu der (nicht allein aussagekräftigen) quantitativen Betrachtung qualitative Kriterien hinzu, so fällt die Bewertung noch dramatischer aus. Einige, zweifelsohne wertvolle, Beiträge beschränken sich auf rein rechtswissenschaftliche Analysen (Bothe 2008; de Wet 2008; Kastner 2010), andere liefern einen guten Überblick, lassen aber eine vertiefte (friedens-)wissenschaftliche Fundierung vermissen. ${ }^{1}$ Daneben liefern gelegentliche Themenhefte skizzenhafte Einführungen in die Materie. ${ }^{2}$ Analysen, die sich intensiver mit der Materie auseinandersetzen, lassen sich allerdings an zwei Händen abzählen. Das Gros dieser lesenswerten Abhandlungen widmet sich anderen Formen internationaler Gerechtigkeitsbestrebungen: Kastner (2007) widmet ihren Beitrag Wahrheitskommissionen, Buckley-Zistel et al. (2010) befassen sich mit Transitional Justice, während Jetschke (2011) und Mayr-Singer (2008) hybride Tribunale und Horelt (2011), Schomburg (2009) und von Braun (2007) das Jugoslawientribunal in den Blick nehmen. Eine vertiefte

1 Däubler-Gmelin (2008); Gutschker (2009); Hankel (2009); Heuwagen (2009); Hummitzsch (2008); van Ooyen (2007).

2 Mittelweg 36 1/2009 zum Thema »Sexuelle Gewalt in kriegerischen Konflikten«; Internationale Politik 1/2010 zum Thema »Völkermord«; Vereinte Nationen 6/2010; Wissenschaft \& Frieden 2/2007 zum Thema »Menschenrechte kontra Völkerrecht?«. 
Auseinandersetzung mit dem Internationalen Strafgerichtshof und seiner aktuellen Tätigkeit findet sich nur bei zwei Autoren: Burchard und Schabas.

Burchard wagt den »Brückenschlag von der Rechts- zur Politikwissenschaft«, indem er »das Völkerstrafrecht als global governance konzipiert« (Burchard 2008: 74). Er wirft dabei Schlaglichter auf Phänomene der modernen Praxis internationaler Strafverfolgung, die weiterer wissenschaftlicher Untersuchung bedürfen. So weist er u.a. auf die »Externalisierung und auch Privatisierung der völkerstrafrechtlichen Ermittlungstätigkeit« (Burchard 2008: 81) und den prägenden Einfluss von Nichtregierungsorganisationen auf die Fortentwicklung des Völkerstrafrechts hin (Burchard 2008: 80). Schabas liefert in seinem Beitrag »O Brave New World: The Role of the Prosecutor of the International Criminal Court" (Schabas 2008) eine Fundamentalkritik der bisherigen Anklagetätigkeit und weist dabei richtigerweise auf die besondere Machtfülle des derzeitigen Amtsinhabers Luis Moreno-Ocampo hin. Dabei zeigt Schabas präzise auf, dass die Entwicklung der IStGH-Tätigkeit keineswegs durch das Gründungsstatut vorgezeichnet ist, sondern durch die Umstände, Personen und Interessen verschiedener Beteiligter geprägt wird. So stellt Schabas im Hinblick auf die strafrechtlichen Ermittlungen gegen den sudanesischen Präsidenten fest: »It was a policy decision by an individual prosecutor« (Schabas 2008: 11).

\section{Internationale Strafverfolgung - mehr als nur Rechtsanwendung}

Internationale Strafverfolgung ist mehr als Aktenarbeit und Rechtsdogmatik; das Handwerkszeug des Rechtskundigen vermag daher die Komplexität der Materie nur ungenügend zu erfassen. Es vermag keine Auskunft darüber zu geben, wie die Bürgerkriegsgesellschaften in Uganda oder Kongo und deren politische Eliten auf internationale Strafverfolgungsbemühungen reagieren, wie und ob diese zur Versöhnung oder Befriedung beitragen oder wie sich lokale Akteure die internationalen Strafprozesse zu Nutzen machen. Es kann nicht klären, was den UN-Sicherheitsrat und seine (ständigen) Mitglieder dazu bewegt, in den herkömmlichen Sanktionskatalog (diplomatische Isolation, Wirtschaftssanktionen, targeted sanctions) die Verweisung an den IStGH aufzunehmen. Das Beispiel Libyen zeigt, wie insbesondere seitens der Westmächte die Internationalisierung der Strafverfolgung betrieben und im gleichen Atemzug als Verhandlungsmasse wieder zur Disposition gestellt wurde. Kaum war der IStGH durch den UN-Sicherheitsrat befasst worden, wurde hinter den Kulissen diskutiert, welcher Staat Gaddafi aufnehmen könnte, um diesem Straffreiheit im Gegenzug für politische Konzessionen zu gewähren. Das juristische Handwerkzeug vermag ebenfalls nicht aufzuzeigen, welche Rolle die nichtstaatliche 
Öffentlichkeit beim Agenda-Setting spielt. So haben sich Nichtregierungsorganisationen wie Amnesty International oder Human Rights Watch zu »Allrounddienstleistern« der internationalen Strafverfolgung entwickelt. Sie tragen Informationen zusammen, bauen medialen Druck auf die politische (westliche) Führungselite auf und liefern juristische Analysen frei Haus.

Selbst die Tätigkeit der Organe des IStGH kann mittels juristischer Instrumentarien nur unzureichend verstanden werden. Sie helfen nicht dabei, nachvollziehen zu können, wie sich der IStGH und seine wichtigsten Repräsentanten auf dem internationalen Parkett positionieren und wie Letztere die zur Verfügung stehenden rechtlichen Interpretationsspielräume nutzen, die das Gründungsstatut gewährt. Dabei lässt sich anhand der bisherigen Praxis deutlich erkennen, dass Chefankläger und Richter in der Lage sind, wichtige Weichen für die Zukunft des IStGH zu stellen.

Schabas (2008) hat bereits auf die Freiheiten des Chefanklägers bei der Auswahl der Fälle hingewiesen. Dies hat enorme Auswirkungen. So war der Chefankläger des IStGH innerhalb kürzester Zeit in der Lage, einen Haftbefehl gegen die libysche Führung zu beantragen. Im Hinblick auf die massenhaft begangenen Verbrechen im Mitgliedsstaat (!) Afghanistan sind hingegen bis heute noch nicht einmal offizielle Ermittlungen eingeleitet worden. Ähnliche Freiheiten stehen dem Chefankläger im Hinblick auf mögliche Verfahrenseinstellungen gemäß Art. 53, bei der Ausgestaltung der Kooperation mit den Vereinten Nationen, der medialen (Selbst-)Darstellung und der Auswahl fallbezogener Anklagestrategien zu. Dabei handelt es sich nicht nur um rein technische Verfahrensfragen, sondern vielmehr um fundamentale Grundentscheidungen, wie z.B.: Sollten Verfahren eingestellt werden, wenn diese nachweislich Friedensbemühungen beeinträchtigen? Sehr konkret stellt(e) sich diese Frage im Zusammenhang mit den Friedensbemühungen in Nord-Uganda. Dort sind nach Ansicht lokaler Geistlicher Friedensverhandlungen mit der Lords' Resistance Army an der drohenden internationalen Strafverfolgung gescheitert. Die Anklagebehörde hat jedoch, aus teilweise schwer nachvollziehbaren Gründen, die möglichen negativen Auswirkungen auf Friedensverhandlungen für rechtlich irrelevant erklärt. Neben der damit zusammenhängenden Peace-vs-Justice-Debatte stellen sich weitere essentielle Fragen, die keiner allein normativen Lösung zugeführt werden können. Ist es beispielsweise sinnvoll, zur Vorbereitung von Verfahren auf geheime Quellen zurückzugreifen und UN-Friedensmissionen zur Beweisgewinnung einzusetzen und so möglicherweise deren Neutralität zu gefährden? Sollte die Anklagebehörde bereits in einem frühen Stadium der Ermittlung die Öffentlichkeit suchen, um die Beantragung (nicht der den Richtern vorbehaltene Erlass) eines Haftbefehls medienwirksam zu verkünden? Was bewegt die Anklage dazu, bestimmte Delikte in den Vordergrund der Ermittlung zu rücken? Warum gerieten 
beispielsweise die Vorwürfe der Massenvergewaltigungen im Lubanga-Verfahren in den Hintergrund und warum besteht die Anklage darauf, den Haftbefehl gegen den sudanesischen Präsidenten Omar al-Bashir auf den (politisch aufgeladenen) Vorwurf des Völkermords zu erstrecken, obwohl die rechtliche Einordnung der Taten im momentanen Verfahrensstadium vollkommen ohne Bedeutung ist? Diese Entscheidungen der Anklagebehörde lassen sich mittels eines juristischen Handwerkszeugs nicht ausreichend verstehen.

Daneben stehen auch den Richtern weitgehende Befugnisse zur Ausgestaltung der IStGH-Tätigkeit zu. So hatten diese zu entscheiden, ob die gewalttätigen Auseinandersetzungen im Zusammenhang mit den kenianischen Wahlen zum Jahreswechsel 2007/2008 vom IStGH strafrechtlich aufgearbeitet (ICC-01/09-19, v. 31-02-2010) und ob sich dieser auch mit Formen organisierter Kriminalität befassen sollte. Mit anderen Worten: Wie weit sollte sich die internationale Gemeinschaft in »interne Angelegenheiten« einmischen? Auch im Lubanga-Verfahren hatten die Richter Position zu beziehen, wurde dort doch mehrfach gegen die Rechte des Angeklagten verstoßen. Die Richter der Verfahrenskammer waren geneigt, den Prozess scheitern zu lassen und den Angeklagten auf freien Fuß zu setzen. Die Berufungskammer war hingegen offensichtlich nicht gewillt, ein Scheitern im ersten Verfahren zuzulassen, und baute der Anklage mehrfach goldene Brücken, so dass das Verfahren weiter geführt werden konnte (vgl. Hiéramente 2010).

Auch in Zukunft werden die Entscheidungsträger des IStGH Realpolitik und Rechtsanwendung unter einen Hut bringen müssen. Ein spannender neuer Streitfall zeichnet sich bereits ab. Die Palästinensische Autonomiebehörde hat den IStGH ersucht, das Handeln israelischer Sicherheitskräfte in Gaza und Westbank auf dessen Strafwürdigkeit hin zu überprüfen. Art. 12 III des Gründungsstatuts sieht vor, dass sich ein »Staat « dem Statut unterwerfen kann, ohne vorher den Status einer Vertragspartei zu beantragen. Richter und Anklage müssen sich dann damit auseinandersetzen, ob Palästina als Staat im Sinne des Statuts anzusehen ist. Auf die politische Brisanz einer derartigen Entscheidung und möglicherweise folgender Strafverfahren braucht hier nicht detailliert eingegangen werden.

\section{Die Friedensforschung wird gebraucht}

Damit sind bereits einige Fragestellungen angedeutet, die von der Friedensforschung zum Anlass für eine vertiefte Auseinandersetzung gemacht werden könnten und sollten. So gilt es, empirische Erkenntnisse zum friedensfördernden, aber auch disruptiven Potential internationaler Strafprozesse zu sammeln und wissenschaftlich aufzuarbeiten. Es gilt zu verstehen, wie Bürgerkriegsgesellschaften die straf- 
rechtliche Aufarbeitung im fernen Den Haag aufnehmen und anerkennen und welchen Einfluss die Fairness des Verfahrens und die Auswahl der Fälle für die Akzeptanz der getroffenen Entscheidungen haben. Ferner gilt es, Bedingungen herauszuarbeiten, die eine stabilisierende Wirkung internationaler Strafverfolgung ermöglichen, steigern oder aber ausschließen. So können Erkenntnisse sowohl für die strategische Ausrichtung des IStGH selbst als auch andere Akteure der Friedensschaffung, z.B. die Vereinten Nationen, gewonnen und die Tätigkeit des IStGH, im Rahmen des normativ Möglichen, optimiert werden. Mögliche Forschungsfragen sind zahlreich und können hier nur skizzenhaft Erwähnung finden. So stellt sich z.B. die Frage, ob die Haftbefehle gegen (verhandlungsbereite) Rebellen Auswirkungen auf Friedensprozesse (Beispiel Uganda) haben und wenn ja, welche. Nur auf Grundlage dieser Erkenntnisse vermag der Chefankläger eine Einstellung eines Verfahrens oder die Vereinten Nationen ein Einfrieren eines solchen gemäß Art. 16 des Rom-Statuts in Erwägung ziehen. Weiter gilt es beispielsweise zu erforschen, welche Rolle internationale Strafverfahren bei (diskursiven) Legitimierungsstrategien für militärische Interventionen spielen (Beispiel Libyen). Zur Beantwortung vergleichbarer Fragestellungen bedarf es des methodischen Instrumentariums der Friedensforschung und des Engagements der Friedensforscher und -forscherinnen. Wie relevante Entscheidungen gefällt werden, wodurch sie beeinflusst werden und wie die Interaktion verschiedener Staaten und internationaler Organisationen bei der Beendigung, Aufarbeitung und Prävention von friedensgefährdenden Systemunrecht verstanden werden kann, ist jedoch derzeit in Deutschland durch die Friedensforschung noch unzureichend untersucht. Ein besseres Verständnis des Verhältnisses von Frieden zu Recht und der Funktionsbedingungen für Friedens- und Versöhnungsprozesse ist für Völker(straf)rechtler jedoch ebenso unerlässlich wie die Beherrschung des juristischen Handwerkszeugs. Die Ausgestaltung und Anwendung völkerrechtlicher Normenkomplexe sollte in Kenntnis von Konsequenzen und Implikationen erfolgen, um die Entfaltung des friedensfördernden Potentials des IStGH und vergleichbarer Institutionen zu gewährleisten; eine Kenntnis, welche die Friedensforschung mit ihrem Fokus auf Konfliktursachen, -prävention und -regelung zu liefern in der Lage ist. Berührungsängste müssen daher überwunden werden und eine stärkere (friedens-)wissenschaftliche Auseinandersetzung mit dem IStGH und seinem Wirken sollte ermutigt werden. Die neue Zeitschrift für Friedensund Konfliktforschung sollte fortan die Gelegenheit bieten, sich mit der Tätigkeit internationaler Strafverfolgungsbehörden, insbesondere des IStGH, ausführlicher, kritisch und interdisziplinär auseinanderzusetzen. 


\section{Literatur}

Bothe, Michael 2008: Complementarity. Ensuring Compliance with International Law through Criminal Prosecutions. Whose Responsibility?, in: Friedenswarte 83: 4, 59-72.

Buckley-Zistel, Susanne/Mieth, Friederike/Viebach, Julia 2010: Transitional Justice und Entwicklung in Afrika, in: Friedenswarte 85: 1-2, 83-109.

Burchard, Christoph 2008: Völkerstrafrecht als global governance, in: Friedenswarte 83: 4, 73-113.

Däubler-Gmelin, Herta 2008: Internationaler Strafgerichtshof. Erfolge und Defizite, in: Blätter für deutsche und internationale Politik 7/2008, 78-84.

de Wet, Erika 2008: The Relationship between the International Criminal Court and Ad hoc Criminal Tribunals. Competition or Symbiosis?, in: Friedenswarte 83: 4, 33-57.

Gutschker, Thomas 2009: Die Hölle vor Gericht, in: Internationale Politik 64: 4, 68-75.

Hankel, Gerd 2009: Recht im Weltmaßstab. Warum der Erlass des Haftbefehls gegen Omar al-Bashir richtig ist, in: Mittelweg 36 18: 2, 77-88.

Heuwagen, Marianne 2009: Niemand steht über dem Gesetz, in: Internationale Politik 64: 4, 76-80.

Hiéramente, Mayeul 2010: Ein faires Verfahren für Kriegsverbrecher - Grenzen der Informationsbeschaffung und der Fall Lubanga, in: Humanitäres Völkerrecht Informationsschriften 23: 1, 32-39.

Horelt, Michel-André 2011: Durch Recht oder Symbolik zur Versöhnung? Ein Vergleich der Versöhnungswirkung des Internationalen Strafgerichtshofs für das ehemalige Jugoslawien (ICTY) und politischer Entschuldigungen im ehemaligen Jugoslawien, in: Friedenswarte 86: 1-2, 131-154.

Hummitzsch, Thomas 2008: Das Kambodscha-Tribunal. Späte Gerechtigkeit?, in: Internationale Politik und Gesellschaft: 4/2008, 87-104.

Jetschke, Anja 2011: Der Kaiser hat ja gar keine Kleider an! Strafverfolgung durch hybride Tribunale, in: Friedenswarte 86: 1-2, 101-130.

Kastner, Fatima 2007: Weder Wahrheit noch Recht. Zur Funktion von Wahrheitskommissionen in der Weltgesellschaft, in: Mittelweg 36 16: 3, 31-50.

Kastner, Philipp 2010: Africa. A Fertile Soil for the International Criminal Court?, in: Friedenswarte 85: 1-2, 131-159.

Mayr-Singer, Jelka 2008: Hybridgerichte - eine neue Generation internationaler Strafgerichte, in: Vereinte Nationen 56: 2, 68-72 und 6, 258-262. 
Schabas, William 2008: O Brave New World. The Role of the Prosecutor of the International Criminal Court, in: Friedenswarte 83: 4, 11-31.

Schomburg, Wolfgang 2009: Wahrheitsfindung im Internationalen Gerichtssaal. Die Ad-Hoc-Strafgerichtshöfe für das ehemalige Jugoslawien und Ruanda, in: Vereinte Nationen 57: 1, 3-9.

van Ooyen, Robert Chr. 2007: Politische Bedingungen einer effektiven internationalen Strafgerichtsbarkeit, in: Internationale Politik und Gesellschaft 3/2007, 23-35.

von Braun, Leonie 2007: Der Strafgerichtshof für das ehemalige Jugoslawien. Mehr Errungenschaften als Versäumnisse, in: Vereinte Nationen 55: 4, 148-152.

\section{Der Autor}

Mayeul Hiéramente, Max-Planck-Institut für ausländisches und internationales Strafrecht, Research School on Retaliation, Mediation and Punishment

E-Mail:m.hieramente@mpicc.de 